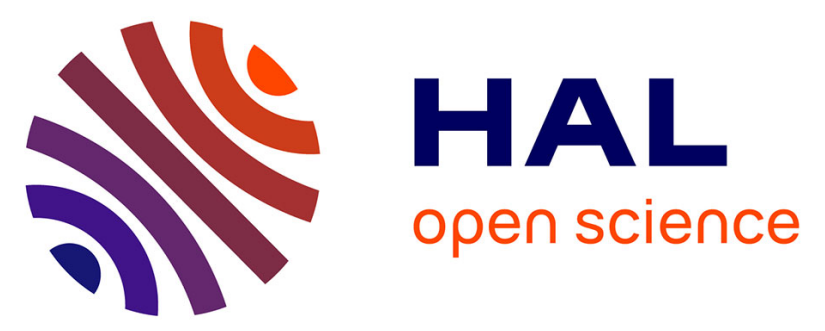

\title{
BD2I : Normes sur l'identification de 274 images d'objets et leur mise en relation chez l'enfant français de 3 à 8 ans
}

Christine Cannard, Françoise Bonthoux, Agnès Blaye, Nelly Scheuner, Anne-Caroline Schreiber, Jacques Trinquart

\section{- To cite this version:}

Christine Cannard, Françoise Bonthoux, Agnès Blaye, Nelly Scheuner, Anne-Caroline Schreiber, et al.. BD2I : Normes sur l'identification de 274 images d'objets et leur mise en relation chez l'enfant français de 3 à 8 ans. Annee Psychologique, 2006, 106 (3), pp.375-396. 10.4074/s0003503306003034 . hal-00153698

\section{HAL Id: hal-00153698 \\ https://hal.science/hal-00153698}

Submitted on 25 Apr 2013

HAL is a multi-disciplinary open access archive for the deposit and dissemination of scientific research documents, whether they are published or not. The documents may come from teaching and research institutions in France or abroad, or from public or private research centers.
L'archive ouverte pluridisciplinaire HAL, est destinée au dépôt et à la diffusion de documents scientifiques de niveau recherche, publiés ou non, émanant des établissements d'enseignement et de recherche français ou étrangers, des laboratoires publics ou privés. 


\title{
BD2I : Normes sur l'identification de 274 images d'objets et leur mise en relation chez l'enfant français de 3 à 8 ans
}

\author{
Christine Cannard ${ }^{\star 1}$, Françoise Bonthoux ${ }^{1}$, Agnès Blaye ${ }^{2}$, \\ Nelly Scheuner ${ }^{1}$, Anne-Caroline Schreiber ${ }^{3}$ Jacques Trinquart ${ }^{4}$ \\ ${ }^{1}$ Laboratoire de Psychologie et NeuroCognition, \\ Université Pierre Mendés France, CNRS UMR 5105 \\ ${ }^{2}$ Centre de Recherche en Psychologie du Langage, \\ de la Connaissance et de l'Émotion, Université de Provence \\ ${ }^{3}$ Cellule TICE (Technologies de l'Information \\ et de la Communication pour l'Éducation), IUFM, Grenoble \\ ${ }^{4}$ CRI (Centre de Ressources Informatiques), \\ Université Pierre Mendés France, Grenoble
}

\section{RÉSUMÉ}

La base de données informatisée BD2I fournit les premières normes françaises chez l'enfant pour 274 images d'objets (150 de Snodgrass et Vanderwart, 1980). L'identification et la dénomination correctes de l'objet ainsi que l'identification d'associations taxonomiques et thématiques ont été testées entre 3 et 8 ans, la justification et la force de ces associations entre 4 et 8 ans. Toutes ont été recueillies auprès de 80 enfants par tranche d'âge. La similarité perceptive a été jugée par 40 adultes. Ces données normatives sont nécessaires puisque l'enfant est moins performant en dénomination que l'adulte et que la représentation qu'il se fait des relations entre objets differe de celle de l'adulte.

BD2I: Norms on identification of 274 pictures of objects and their relation with French children aged between 3 to 8 years-old ABSTRACT

The data base BD2I provides the first French norms for children of 274 pictures of objects (150 from Snodgarss and Vanderwart, 1980). Correct picture identification and naming, and identification of taxonomic and thematic associations were evaluated between 3 and 8 years. Verbal justification and strength of the associations were assessed between 4 and 8 years. All norms were collected from 80 children by age. Visual similarity was judged by 40 adults. These normative data are necessary because children are less efficient than adults in naming and also because their representations of objects relations differ from those of adults.

\footnotetext{
* Correspondance : Laboratoire de Psychologie et Neurocognition, Bâtiment des Sciences de l'Homme et des mathématiques, Domaine Universitaire de St-Martin d'Hères, 1251 Avenue Centrale, BP 47, 38040 Grenoble Cedex 9. Remerciements : La constitution de la base a pu être faite grâce à la subvention du Ministère de la Recherche, en réponse à l'ACI Cognitique 2000 « Nouvelles technologies et cognition ». Les auteurs remercient les inspecteurs de l'Éducation nationale, les directeurs(trices) d'écoles, les enseignants, ainsi que les nombreux parents et enfants de leur coopération.
} 


\section{INTRODUCTION}

Cet article présente BD2I, une base de données informatisée d'images qui fournit les premières normes françaises d'identification des objets et des relations qu'ils entretiennent, recueillies sur une population d'enfants âgés de 3 à 8 ans. L'objectif est de remédier à l'absence de telles normes en langue française afin de fournir un outil méthodologique rigoureux aux chercheurs, évitant ainsi les multiples tests préalables à la constitution des protocoles expérimentaux utilisant du matériel imagé (voir par exemple Blaye \& Bonthoux, 2001; Scheuner, Bonthoux, Cannard, \& Blaye, 2004; Waxman \& Namy, 1997). Ces normes sont accessibles sur le Web et concernent 274 images d'objets dessinés au trait, dont 150 images de Snodgrass et Vanderwart (1980), l'ensemble d'images le plus utilisé dans le monde.

De nombreuses recherches en psychologie cognitive font appel à des tâches expérimentales reposant sur du matériel imagé. Dans les études sur le développement des catégories et des concepts chez l'enfant, qui nous intéressent plus particulièrement, les auteurs utilisent souvent des épreuves d'appariement en choix forcé. Dans celles-ci, on présente à l'enfant plusieurs images, une cible et des objets qui ont été choisis pour partager avec la cible diverses relations catégorielles (par exemple, thématique, taxonomique ou perceptive). L'identification des images isolées est évaluée en général lors de pré-tests. Mais, ni le degré de familiarité des exemplaires de chaque catégorie, des associations entre objets ou des catégories elles-mêmes, ni la justification verbale ou la force des associations proposées ne sont contrôlés dans la majorité des recherches. Les associations entre images sont choisies a priori par l'expérimentateur. On évalue uniquement si l'enfant a bien compris la tâche en le familiarisant avec quelques associations avant le début de l'expérimentation proprement dite. Afin d'augmenter la validité des recherches concernant la structuration des connaissances chez l'enfant dans les différents domaines sémantiques, les chercheurs ont besoin de normes portant à la fois sur les images isolées et les relations qu'entretiennent ces images. Au total, six indices normatifs très différents sont disponibles dans BD2I à chaque âge entre 3 et 8 ans. Étant donné la quantité considérable d'informations que représentent ces six indices, il n'est pas envisageable de présenter ici le résultat des analyses de données. Plusieurs articles permettent d'ores et déjà de répondre à des hypothèses de recherche précises (Cannard, Blaye, Scheuner, \& Bonthoux, 2005). Le présent article est centré sur la présentation des épreuves ainsi que la justification de leur choix à partir d'objectifs de recherche. 


\section{NORMES EXISTANTES}

Durant ces dix dernières années de très nombreuses normes de dénomination d'images ont été publiées. En revanche, à notre connaissance, aucune étude normative n'a porté sur le développement de la connaissance des relations conceptuelles entre objets chez l'enfant.

\section{Normes sur les images d'objets}

Ces dernières années, des chercheurs ont recueilli des normes sur des caractéristiques propres aux images (comme la complexité visuelle) et sur les labels modaux associés (comme la fréquence dans la langue, l'âge d'acquisition, ou la valeur d'imagerie). Les images les plus utilisées sont celles de Snodgrass \& Vanderwart (1980) pour lesquelles ont été collectées les premières données normatives sur des adultes américains. Il est apparu très vite nécessaire de valider ces images sur des populations adultes de communautés culturelles et/ou linguistiques différentes (Alario \& Ferrand, 1999; Barry, Morrison, \& Ellis, 1997; Bates, Burani, D’Amico, \& Barca, 2001; Bates et al., 2003; Cuetos, Ellis, \& Alvarez, 1999; Dell' Acqua, Lotto, \& Job, 2000; Kremin et al., 2003; Martein, 1995 ; Pind, Jonsdottir, Gossurardottir, \& Jonsson, 2000; Sanfeliu \& Fernandez, 1996; Szekely et al., 2003; Van Schjagen, Tamsa, Bruggemann, Jackson, \& Michon, 1983 ; Vitkovitch \& Tyrrell, 1995). Il est peu probable que ces données validées chez l'adulte soient pertinentes dès lors que l'on s'intéresse à des populations d'enfants. Or, peu de recherches à notre connaissance ont recueilli des normes chez l'enfant (Berman, Friedman, Hamberger, \& Snodgrass, 1989; Chainay, Rosenthal, \& Goldblum, 1998; Chalard, Bonin, Méot, Boyer, \& Fayol, 2003; Cycowicz, Friedman, Rothstein, \& Snodgrass, 1997). Berman et al. (1989) ont montré que les performances en dénomination des enfants anglais de 8 et 10 ans étaient équivalentes à celles des adultes dans $84 \%$ des cas. Cycowitz et al. (1997) ont révélé, quant à eux, de fortes différences entre les enfants de 6 ans et les adultes: le nom de l'image diverge dans près de la moitié des cas, plus d'un tiers des réponses sont du type «ne connaît pas le nom de l'objet » ou «ne connaît pas l'objet» et enfin les enfants donnent beaucoup plus de réponses alternatives que les adultes. Ces données normatives ont été confirmées par les résultats des études développementales à échantillons plus réduits. Les performances des enfants s'améliorent avec l'âge, dans le sens d'une plus grande rapidité et d'une plus grande exactitude dans les réponses (Clark \& Johnson, 1994; D’Amico, Devescovi, \& Bates, 2001; 
Wiegel-Crump \& Dennis, 1986). Les omissions diminuent, les bonnes réponses augmentent et les réponses erronées sont de plus en plus souvent liées sémantiquement plutôt que perceptivement à l'image.

L'ensemble de ces résultats montre que si les réponses de l'enfant de 8 ans sont très proches de celles de l'adulte, ce n'est pas le cas chez l'enfant plus jeune qui commet beaucoup d'erreurs de dénomination.

\section{Normes sur les relations entre objets}

En ce qui concerne les normes sur les relations entre objets, les seules études existantes sont issues de la tâche d'association de mots. Par exemple, des normes ont été publiées récemment pour les adultes français (Ferrand \& Alario, 1998) et les enfants français de 9, 10 et 11 ans (De La Haye, 2003). Cette épreuve évalue la fréquence d'association entre concepts verbaux dans la langue mais ne fournit pas d'informations quant à la connaissance des types de relations conceptuelles maîtrisées par les enfants.

L'absence de normes relatives à l'identification d'associations chez l'enfant français rend les contrôles expérimentaux difficiles et limite fortement les conclusions sur les processus cognitifs sous-jacents. Les normes sont en effet nécessaires pour ne pas confondre les différentes variables en jeu. Pour les études développementales du processus de catégorisation par exemple, elles permettent d'interpréter les différences inter-âges, en termes de différences dans les fonctions cognitives en contrôlant le niveau de connaissances lexicales. Ces normes relatives à l'objet lui-même mais aussi aux catégories d'objets et aux relations entre les objets ont été recueillies à chaque âge auprès d'un grand nombre d'enfants.

\section{QUE MESURE BD2I ?}

Nous avons recueilli pour 274 images d'objets, dont 150 tirées de Snodgrass and Vandewart (1980), des normes concernant a) les labels associés aux images (pourcentages d'identification du concept à partir de l'image et de dénomination correcte) et b) les mises en relation des objets (pourcentages d'associations taxonomiques et thématiques identifiées, de justifications correspondantes, score moyen de force d'association et de similarité perceptive). Parallèlement aux normes recueillies sur les images, BD2I donne aussi des normes sur la typicalité des exemplaires de 
56 catégories taxonomiques et thématiques, évaluée à partir d'une épreuve de fluence verbale.

Pour plus de clarté, seront décrits, dans un premier temps, la population participante et le choix des stimuli communs à toutes les tâches et, dans un deuxième temps, les épreuves les unes après les autres. Nous indiquons d'abord l'intérêt théorique justifiant le choix de chaque épreuve et décrivons ensuite la méthodologie associée (population, matériel et procédure expérimentale).

\section{Population}

Chaque item (image isolée ou images associées) a été évalué, dans chaque tâche, chez 80 enfants sur 6 niveaux scolaires : à l'école maternelle, petite section ( 3 ans environ), moyenne section ( 4 ans) et grande section ( 5 ans) et à l'école élémentaire, cours préparatoire ( 6 ans), cours élémentaire $1^{\text {re }}$ année ( 7 ans) et cours élémentaire $2^{\mathrm{e}}$ année ( 8 ans), permettant ainsi une analyse développementale. Un peu plus de neuf mille enfants âgés de 2 ans 9 mois à 8 ans 11 mois des agglomérations de Grenoble et d'Aix-enProvence ont participé au projet. Chaque enfant n'a été confronté qu'à une tâche et n'a répondu qu'à un nombre limité d'items pour éviter les effets de fatigue. Les enfants étaient tous francophones. Les écoles se situaient dans des quartiers moyennement favorisés à très favorisés. ${ }^{1}$

\section{Description des stimuli}

\section{Objets isolés}

Un travail de sélection de l'ensemble des objets représentés dans la banque a été réalisé au préalable. Nous avons choisi essentiellement des objets qui entrent dans la constitution du matériel expérimental des études en catégorisation. La plupart sont des objets pour lesquels d'autres normes sont déjà disponibles, majoritairement chez l'adulte (Alario \& Ferrand, 1999; Barry et al., 1997; Bates et al., 2001; Bates et al., 2003; Cuetos et al., 1999; Cycowicz et al., 1997; Dell' Acqua et al., 2000; Kremin et al., 2003; Martein, 1995; Pind et al., 2000; Sanfeliu \& Fernandez, 1996; Snodgrass \& Vanderwart, 1980; Szekely et al., 2003; Van Schjagen et al., 1983; Vitkovitch \& Tyrrell, 1995).

\footnotetext{
${ }^{1}$ Les accords des inspecteurs de l'Éducation Nationale, des directrices et directeurs des écoles fréquentées et des parents des élèves participants ont été demandés préalablement.
} 


\section{Relations entre objets}

Nos choix relatifs aux associations entre objets ont été déterminés par plusieurs considérations théoriques et par des objectifs de recherche fondamentale. La sélection des items a pris appui sur les études réalisées antérieurement chez les jeunes enfants (par exemple Baldwin, 1992; Blaye, Bernard-Peyron, \& Bonthoux, 2000; Blaye \& Bonthoux, 2001; Golinkoff, Shuff-Bailey, Olgin, \& Ruan, 1995; Imai, Gentner, \& Uchida, 1994; Lucariello, Kyratzis, \& Nelson, 1992; Markman \& Hutchinson, 1984; Rosch, Mervis, Gray, Johnson, \& Boyes-Braem, 1976; Sell, 1992; Smiley \& Brown, 1979; Waxman \& Namy, 1997). Une liste des catégories les plus souvent utilisées a d'abord été établie. Ensuite pour ces catégories, des objets destinés à servir de "cibles" pour les expériences d'appariement incluant divers associés ont été sélectionnés. Nous voulions qu'à chaque cible correspondent deux associés taxonomiques et deux associés thématiques. Les relations taxonomiques regroupent des objets de même sorte, possédant donc des propriétés communes et appartenant à une même catégorie sémantique (par exemple les voitures ou les véhicules), tandis que les relations thématiques regroupent des objets de diverses sortes, faisant partie d'une même scène ou d'un même événement de la vie quotidienne et entretenant donc des liens de proximité spatio-temporelle (par exemple, le bol, la tartine et le chocolat qui sont tous utilisés lors du petit-déjeuner). Les associations proposées dans le site correspondent donc à ces deux types de relations en proportions équivalentes. Il faut signaler que la moitié des associations taxonomiques concerne des catégories contextualisées : celles-ci sont composées d'éléments substituables d'un contexte donné, comme les animaux de la ferme ou les meubles de la cuisine ; ces catégories sont appelées «slot-filler» par Nelson (1983, 1986), c'est-à-dire «pour remplir les cases (d’un schéma)». L'autre moitié correspond à des catégories sémantiques indépendantes des contextes spécifiques (comme les catégories des véhicules ou des aliments).

Un des objectifs étant l'étude des représentations catégorielles en fonction du domaine de connaissances (par exemple objets naturels et objets fabriqués) (voir Bonthoux, Berger, \& Blaye, 2004), le choix des associés a aussi été en partie déterminé par un objectif de représentativité des catégories dans ces deux domaines. À ce jour, la base fournit des normes pour 20 cibles, chacune testée avec 4 associés dans 4 épreuves différentes.

\section{Choix des images}

Une fois cette liste établie, nous avons recherché l'image pouvant s'associer à chaque label lexical en nous appuyant sur les recherches expérimentales disponibles. Nous avons, dans un premier temps, choisi 
les images que notre équipe utilise habituellement, en privilégiant celles le plus souvent utilisées dans la littérature, à savoir celles de Snodgrass et Vanderwart (1980), celles de Berman (1989) et celles de Cycowicz (1997). Nous avons ensuite fait appel à un graphiste pour la constitution de nouvelles images. Au final, la liste comprend 274 objets dessinés au trait noir sur fond blanc. Cent cinquante proviennent de Snodgrass \& Vanderwart (1980), 22 de Cycowicz \& al. (1995) et 102 ont été nouvellement introduits et dessinés par nos soins. Dans la banque, nous avons conservé le principe de numérotation des images de Cycowicz \& al. (1995) : les images de Snodgrass \& Vanderwart (1980) ainsi que celles de Cycowicz \& al. (1995) conservent le numéro des articles originaux (entre 1 et 260 et entre 261 et 399, respectivement, même si elles ne sont pas toutes utilisées) ; les nôtres sont numérotées à la suite (de 400 à 501). La taille des images a été modifiée de sorte que chacune d'entre-elles soit inclue dans un carré de $5 \mathrm{~cm} \times 5 \mathrm{~cm}$.

\section{Description des tâches}

Toutes les épreuves ayant permis la constitution des normes de la base, à l'exception de celle de génération d'exemplaires, ont porté sur un matériel imagé. En outre, à part les tâches de justification de l'association et de jugement de la force de cette association, proposées seulement à partir de 4 ans, et celle de jugement de similarité perceptive effectuée par des adultes, les épreuves ont été proposées à chaque tranche d'âge entre 3 et 8 ans.

\section{Tâche de dénomination d'objet}

Retrouver le nom d'un objet dessiné est un processus complexe. Trois étapes principales, la reconnaissance visuelle, l'activation de l'information sémantique et la sélection lexicale, ont été proposées par la plupart des modèles de dénomination (Humphreys, Riddoch, \& Quinlan, 1988; Johnson, Paivio, \& Clark, 1996; Levelt, 1989). L'identification de l'objet peut être suivie par l'activation d'un ou plusieurs noms en mémoire, mais pas toujours. Un objet peut être reconnu sans activation mentale du nom correspondant (Johnson, 1992; Johnson et al., 1996; Martein, 1995). La plupart des travaux utilisant une épreuve de dénomination à partir d'images étudient donc les processus cognitifs sous-jacents à la dénomination, en analysant quelles sont les caractéristiques de l'image ou du label associé à l'image susceptibles d'affecter le choix d'un label particulier ou le temps mis à le produire. L'âge d'acquisition du nom, par exemple, a été 
reconnu récemment comme le déterminant principal de la dénomination correcte et de la rapidité des réponses chez l'adulte, plutôt que sa fréquence ou sa familiarité (Barry, Hirsh, Johnston, \& Williams, 2001; Bonin, Fayol, \& Chalard, 2001; Caroll \& White, 1973; Gilhooly \& Hay, 1977; Morrison \& Ellis, 1995; Morrison, Ellis, \& Quinlan, 1992; Vitkovitch \& Tyrrell, 1995). La majorité des études évalue subjectivement ce paramètre en demandant aux adultes d'estimer, sur une échelle en 5 ou 7 points, l'âge auquel ils ont acquis tel ou tel mot associé aux images (Barry et al., 1997; Bonin, Chalard, Méot, \& Fayol, 2001, 2002; Bonin, Fayol et al., 2001; Brysbaert, Van Wijnendaele, \& De Deyne, 2000; Caroll \& White, 1973; Cycowicz et al., 1997; Ellis \& Morrison, 1998; Gerhand \& Barry, 1999; Gilhooly \& Gilhooly, 1980; Gilhooly \& Hay, 1977; Kremin, Hamerel, Dordain, De Wilde, \& Perrier, 2000; Morrison, Chappell, \& Ellis, 1997; Morrison et al., 1992). Peu d'études ont recueilli l'âge d'acquisition directement sur des enfants. Celles qui l'ont fait ont cherché à comparer les mesures objectives recueillies chez l'enfant et les mesures subjectives estimées par l'adulte. Les résultats révèlent de fortes corrélations entre les deux mesures, soulignant ainsi le caractère valide de la mesure estimée par l'adulte. Cependant, ils montrent aussi quelques divergences. L'âge d'acquisition objectif est moins corrélé avec la fréquence du mot ou sa familiarité que l'âge d'acquisition estimé par l'adulte. Les auteurs suggèrent donc qu'il est toujours préférable d'utiliser les mesures objectives quand elles sont disponibles, pour éviter l'impact trop fort de la fréquence du mot ou de sa familiarité (Barry et al., 1997; Bonin, Chalard et al., 2001; Chalard et al., 2003; Ellis \& Morrison, 1998; Morrison et al., 1997; Morrison \& Ellis, 1995; Morrison et al., 1992; Pind et al., 2000). Généralement, l'âge d'acquisition du mot est défini par l'âge auquel $75 \%$ des enfants ont pu dénommer correctement l'objet. L'originalité de notre travail est de donner une mesure objective de l'âge d'acquisition à partir de 3 ans, évaluée sur un grand échantillon d'enfants français.

Participants Quatre vingt enfants de chaque tranche d'âge ont dénommé chaque item, mais pour limiter la lourdeur du recueil de données, nous avons considéré que les images correctement dénommées par au moins $80 \%$ des enfants de grande section de maternelle l'étaient aussi par les enfants plus âgés. En conséquence, seules 135 images sur 274 ont été montrées aux enfants de l'école élémentaire. Au total, 1440 enfants ont participé à cette tâche, soit 960 de 3 à 5 ans ( 4 listes - voir procédure $-{ }^{\star} 80$ enfants ${ }^{\star} 3$ tranches d'âges) et 480 de 6 à 8 ans ( 2 listes - voir procédure $-{ }^{*} 80$ enfants ${ }^{*} 3$ tranches d'âges).

Matériel À partir des stimuli sélectionnés, 34 planches de 8 dessins d'objets ont été constituées (plus une planche de 2 dessins seulement). Quatre listes ont ensuite été créées dans lesquelles apparaissent soit 8 , soit 9 planches de 8 dessins. 
Procédure En fonction de l'âge, la passation individuelle a duré de 10 à 20 minutes et s'est faite en une ou deux sessions. L'ordre des planches à l'intérieur de chaque liste est aléatoirement défini par l'expérimentateur ainsi que l'ordre des images pointées.

La consigne est identique à celle donnée par Cycowicz et al. (1997). Après que la première image soit pointée par l'expérimentateur, il est demandé à l'enfant d'identifier cet objet ( "peux-tu me dire quel est ce dessin?») ou de signaler directement s'il ne le connaît pas. En cas d'absence de réponse et dans le but de savoir si, en dépit de toute dénomination, l'enfant parvient malgré tout à identifier l'objet, des précisions sur la fonction de l'objet ou encore ses rencontres éventuelles sont demandées par l'expérimentateur. Toutes les réponses brutes des enfants étaient saisies telles quelles.

\section{Critère d'analyse}

a) Pourcentage de dénomination

Il s'agit du pourcentage d'enfants dénommant correctement l'image. Ainsi, pour éviter toute erreur d'interprétation dans le cas où la dénomination des enfants diverge de celle des adultes, deux juges ont dépouillé séparément une trentaine de protocoles. Dans les cas ambigus, les décisions ont respecté strictement les critères énumérés ci-dessous :

- La dénomination des objets se fait généralement au niveau de base (Rosch et al., 1976), ce niveau variant selon les taxonomies. Par exemple, dans la catégorie des animaux, le niveau de base correspond généralement au niveau de l'espèce pour les mammiferes (chien, cheval) mais à celui de la classe englobante pour les poissons ou les oiseaux. De ce fait dans certains cas, plusieurs réponses ont été acceptées comme des dénominations correctes: par exemple, moineau et oiseau, voilier et bateau ou encore sapin et arbre.

- Certaines abréviations, étant devenues très courantes dans le langage oral de l'enfant mais aussi de l'adulte, ont été acceptées comme, par exemple, télé pour télévision, frigo pour réfrigérateur, vélo pour bicyclette.

- Certaines images renvoyant à plusieurs noms possibles, les synonymes ont été acceptés : par exemple bus et car, ou pot d'eau, broc à eau, pichet, et carafe.

Ces critères de dépouillement sont toujours indiqués dans la base lors de l'énoncé des pourcentages de dénomination.

\section{b) Pourcentage d'identification}

Dans la mesure où de nombreuses images ont été décrites par les enfants très jeunes par des périphrases, le pourcentage d'identification du concept n'impliquant pas sa dénomination a également été calculé. Lorsqu'une réponse autre que le nom était donnée (périphrase), nous avons donc 
cherché dans le dictionnaire Le Robert la définition de chaque mot afin d'établir les critères de dépouillement quant à l'identification de l'objet. Pour être considérées comme des identifications correctes, les productions verbales non attendues devaient permettre de différencier l'objet représenté des objets proches de la même catégorie. " Pour mettre sur la tête à la neige » ne suffit pas, par exemple, à confirmer l'identification de la "cagoule" dans la mesure où cela renvoie aussi à bonnet, alors que lorsqu'un enfant dit " pour enlever l'eau des pâtes » pour l'image de la passoire, il signifie qu'il connaît l'objet mais qu'il ne connaît pas son nom.

Le score d'identification indiqué sur le site Web inclut le pourcentage de dénomination auquel s'ajoute celui des identifications correctes par périphrases. La différence de pourcentage entre l'identification et la dénomination correspond à la proportion de réponses indiquant une connaissance des objets mais pas des termes permettant de les dénommer.

En résumé, sur le site Web, chaque image apparaît avec un pourcentage de dénomination correcte (si plusieurs noms sont considérés comme corrects, ils sont mentionnés en note) ainsi qu'avec un pourcentage d'identification (égal ou supérieur au précédent) à chaque âge entre 34 ans et 5-6 ans. Pour les classes d'âge de 6-7 ans à 8-9 ans, la mention " inféré » apparaît pour ces deux normes sur une partie des images, indiquant qu'elles ont été dénommées par au moins $80 \%$ des enfants de maternelle, et donc, n'ont pas été testées sur les enfants plus âgés, pour ces deux critères.

\section{Tâche d'identification des associations entre objets}

Les travaux de Walsh, Richardson et Faulkner (1993) ou d'Osborne \& Calhoun (1998) chez l'enfant et, plus récemment, ceux de Lin et Murphy (2001) chez l'adulte ont montré que les objets choisis pour instancier les relations catégorielles jouaient un rôle majeur dans les choix d'appariement. Les travaux de Lin et Murphy, reprenant ceux de Smiley et Brown (1979) révèlent que l'on peut, selon les items choisis, faire basculer la "préférence conceptuelle » vers les relations taxonomiques ou thématiques. De même, dans des tâches de jugement de la similarité ou de l'association thématique, les performances des adultes sont influencées par les caractéristiques des stimuli (Wisnieski \& Bassok, 1999). Dans certaines recherches chez l'enfant, le niveau d'identification des associations proposées doit impérativement être contrôlé. C'est le cas par exemple de l'étude de la flexibilité catégorielle (Blaye \& Bonthoux, 2001). La flexibilité catégorielle correspond à la capacité à envisager diverses relations pour un objet donné (par exemple pomme - couteau et pomme - 
banane). De faibles scores de flexibilité peuvent résulter soit d'une méconnaissance de certaines associations proposées, soit d'une véritable difficulté à envisager deux relations différentes pour le même objet. L'objectif de la tâche d'identification des associations est de déterminer quelle paire d'objets est reconnue comme partageant une relation catégorielle, en l'absence d'interférence provenant d'une seconde relation.

Matériel Des planches de 4 objets dessinés au trait ont été utilisées. Dans la partie supérieure de la planche, se trouve l'objet cible et dans la partie inférieure apparaissent les 3 images parmi lesquelles une seule partage une relation catégorielle, taxonomique ou thématique, avec la cible ; les deux autres objets sont donc des distracteurs, non associés à la cible. Chaque cible a été proposée avec 4 associés différents: 2 relations thématiques et 2 relations taxonomiques, l'une du niveau surordonné ( 2 animaux) et l'autre de type slot-filler ( 2 animaux de la ferme ou domestiques). Dans un souci de comparabilité, ce sont les mêmes distracteurs qui ont été utilisés pour les 4 associations relatives à une même cible. Le nom de ces distracteurs est mentionné sur le site. Les planches ont été réparties en 2 listes, comprenant chacune 40 associations. Dans chaque liste, chaque cible apparaît une seule fois, soit avec un associé taxonomique, soit avec un associé thématique.

Procédure Chaque enfant n'est confronté qu'à une seule liste ${ }^{1}$. La procédure est la même que pour la tâche de dénomination; seule la consigne change. On demande à l'enfant de bien réfléchir et de dire "quelle image parmi les 3 va bien avec la cible». Aucune justification n'est demandée.

Critère d'analyse : pourcentage d'identification des associations Il s'agit, pour chaque niveau d'âge, du pourcentage d'enfants ayant reconnu l'association entre la cible et l'objet parmi des distracteurs.

\section{Tâche de justifications des associations entre objets}

La tâche d'appariement ne met en relation que deux images, contrairement à celle de tri libre qui réunit généralement un plus grand nombre d'objets par groupe. Dès lors, la sélection d'une paire d'images reste souvent ambiguë quant à la représentation qui sous-tend l'association et ce, particulièrement chez les jeunes enfants (Blaye et al., 2000). La prise en compte des justifications verbales fournies peut donc permettre de mieux comprendre la représentation conceptuelle évoquée par l'enfant, représentation qui ne correspond pas nécessairement à celle pour laquelle l'adulte a proposé le matériel. Les auteurs ayant tenté de prendre en considération ces productions linguistiques ont souligné les " distorsions entre la nature des choix opérés par les enfants et leurs justifications verbales » (Chemlal, 2000, p 138). En particulier, les associations supposées relever d'une appartenance à une catégorie taxonomique commune s'avèrent parfois

${ }^{1} 2$ listes ${ }^{\star} 80$ enfants ${ }^{\star} 6$ tranches d'âge $=960$ enfants vus. 
justifiées par un lien thématique (Blaye et al., 2000; Bonthoux \& Blaye, 1999; Lucariello et al., 1992; Sell, 1992; Walsh et al., 1993)

Matériel Les 80 associations sélectionnées précédemment pour l'épreuve de reconnaissance étaient proposées à un nouvel échantillon d'enfants. Les distracteurs ont été enlevés pour ne faire apparaître que des paires d'objets : la cible et l'associé. Les deux mêmes listes que précédemment ont donc été constituées, sans les distracteurs.

Procédure Chaque enfant n'était interrogé que sur une liste. Pour chaque paire d'images, la consigne était la suivante: «tu vois, certaines personnes ont mis ensemble ces deux objets, peux-tu me dire pourquoi ? ». Lorsque l'enfant ne répondait pas, on lui demandait de bien regarder les deux objets pour essayer de trouver le lien qu'ils entretenaient. Toutes les réponses brutes des enfants étaient recueillies.

Critère d'analyse: pourcentage des justifications catégorielles Les justifications verbales ont été classées en trois grands types : taxonomiques, thématiques et perceptives. Une explication est cotée perceptive si l'enfant a cité des propriétés communes liées seulement à l'apparence physique comme la forme ou des parties communes (pour pomme et gâteau : «les deux sont ronds »; pour voiture et vélo : «les deux ont des roues »). Il n'y a pas de difficulté particulière de cotation pour ce type de justification. Nous n'avons cependant pas pris en compte l'énoncé «c'est pareil », jugé insuffisant pour déterminer le type de catégorisation auquel il renvoyait.

En revanche, les cotations des explications comme thématiques ou taxonomiques sont plus délicates. Nous nous sommes basées sur celle préconisée par Lucariello et al. (1992) et Sell (1992), faisant référence aux travaux de Nelson. Selon ces auteurs, pour établir qu'une justification renvoie à une relation thématique, il faut que soit mentionné un lien spatial ou temporel entre les deux objets ou encore une action commune incluant les deux objets (pour gant et ski, « on met des gants, puis on va faire du ski »; pour ballon et vélo : "on va jouer au ballon en vélo »). Par ailleurs, pour établir qu'une justification sous-tend une relation taxonomique, il faut que la substituabilité des deux objets de la paire soit clairement énoncée (pour chien/ poule, "ce sont deux animaux»; pour carotte/fraise, "on les mange tous les deux »). Il faut noter que le choix de ce critère implique que les associés taxonomiques surordonnés («deux vêtements») et ceux limités à un script ne sont pas différenciés («deux vêtements pour aller à la neige»), et sont cotés tous les deux selon une justification taxonomique.

Une fois ces critères mis en place, une liste d'exemples types d'énoncés a été établie. Pour chaque relation, une centaine de protocoles de tous âges a été cotée indépendamment par deux participants au projet. Le pourcentage d'accord étant satisfaisant (97\%), le reste des protocoles a été dépouillé et coté par une des deux personnes seulement.

\section{Tâche d'évaluation de la force des associations entre objets}

Une étude publiée par Scheuner, Bonthoux, Blaye \& Cannard (2004) a mis en évidence l'effet de la force d'association sur les conduites d'appa- 
riement d'images. Les résultats ont montré que a/ pour une même cible, les choix d'appariement sont modulés par la force d'association des associés proposés, b/ les associations taxonomiques renvoient globalement à des forces plus faibles que les associations thématiques, et c/ une mesure des forces d'association est valide dès l'âge de 4 ans. Ainsi, des normes sont nécessaires pour contrôler l'impact de ce facteur sur les performances de catégorisation.

Matériel Les 80 paires d'objets utilisées pour la tâche de justification des associations, réparties là encore en deux listes, étaient présentées à de nouveaux enfants. Pour adapter la tâche à l'âge des enfants, nous avons re-créé l'échelle de mesure utilisée par Scheuner et al. (2004), elle-même inspirée des échelles d'évaluation de la douleur dans le domaine médical. Ainsi, à l'aide d'une planche colorée sur laquelle il est possible de déplacer un curseur, les enfants devaient évaluer, sur une échelle graduée de 0 à 10 , la force d'association entre chaque paire d'objets proposée. Le niveau 0 correspondait à une évaluation faible de l'association proposée alors que le niveau 10 correspondait à une très forte estimation. Ainsi, cette échelle permet $1 /$ des jugements affinés grâce à un passage obligatoire par chacune des valeurs intermédiaires lorsqu'on déplace le curseur, 2/ une meilleure correspondance avec la logique de l'enfant «ce qui est haut est fort (-ement associé) versus ce qui est bas est faible (-ment associé) et enfin, 3/ un maintien de l'attention du fait de la participation active de l'enfant durant le test (manipulation du curseur). De plus, pour rendre ce matériel encore plus attractif, la forme graphique de l'échelle a été améliorée (taille et couleur du curseur, insertion de petits dessins de visages, souriant - niveau 10 de l'échelle - et non souriant - niveau 0 de l'échelle -).

Procédure Du fait de sa difficulté (la compréhension de l'échelle est difficile chez les plus jeunes et la passation est longue), cette épreuve n'a été proposée qu'à partir de 4 ans et n'a été administrée qu'à 40 enfants par tranche d'âge. Il leur était demandé d'estimer la force d'association entre les deux images d'objets présentées : "montre moi avec le petit triangle rouge (le curseur) si les deux images vont très très bien ensemble (10), très bien (7-8), assez bien (4-6), pas trop bien (2-3), un tout petit peu (1) ou pas du tout (0). Au début de la session, plusieurs exemples sont réalisés par l'expérimentateur qui donne des précisions sur les nuances entre chaque niveau de l'échelle. Puis d'autres exemples (essais d'entraînement) sont proposés à l'enfant au cours desquels des explications complémentaires sont éventuellement fournies. Lorsque l'enfant ne comprend pas après de nouvelles explications, l'expérimentateur met un terme à l'expérience (par exemple, ceux qui alternent entre 0 et 10 seulement ou ceux qui donnent le même degré de force à tous les items). Pour les autres, une série de 40 associations leur était proposée.

Critère d'analyse : degré de force d'association entre deux objets Cette norme correspond au score moyen, sur l'échelle de 0 à 10 , obtenu pour chaque paire d'images par les 40 enfants à chaque tranche d'âge. L'écart type est également indiqué. 


\section{Tâche de similarité perceptive}

Lorsque l'on étudie les processus de catégorisation, il convient de se demander sur quelle base les équivalences entre les objets s'établissent. Tant que l'enfant a trop peu d'expérience avec les objets associés qu'on lui présente, il ne peut s'appuyer bien souvent que sur la similarité perceptive (Daehler, Lonardo, \& Bukatko, 1979; Fenson, Cameron, \& Kennedy, 1988; Fenson, Vella, \& Kennedy, 1989). Or, les catégories taxonomiques incluent des objets possédant souvent des attributs physiques communs (avoir une tête, quatre pattes, des angles droits, etc.), la similarité variant selon les catégories et le niveau hiérarchique, tandis que les objets d'une catégorie thématique ne se ressemblent généralement pas (chien, os, niche). Ainsi, lorsque l'on compare des choix d'associations taxonomiques et/ou thématiques, il est essentiel de contrôler ce biais perceptif, en particulier chez l'enfant (Golinkoff et al., 1995; Imai et al., 1994).

Du fait de sa difficulté, cette épreuve n'a pu être proposée qu'à des adultes. On demande en effet un jugement perceptif faisant intervenir le moins possible les aspects sémantiques. Il est très difficile pour l'enfant de faire abstraction de ces connaissances, même si celles-ci sont souvent parcellaires.

Matériel Les 80 paires d'images utilisées pour les épreuves de justification et d'évaluation de la force des associations étaient présentées à des adultes. Répartis là encore en deux listes, les planches étaient présentées à l'intérieur d'un classeur afin de rendre la passation autonome.

Participants Quarante adultes, étudiants en psychologie, ont jugé chaque liste de 40 paires.

Procédure Il s'agit de juger sur une échelle de 1 à 7 la similarité perceptive entre les cibles et les associés, en essayant de ne pas faire intervenir dans les jugements la proximité ou l'éloignement sémantique des objets.

Critère d'analyse: degré de similarité entre deux objets Cette norme correspond au score moyen, sur l'échelle de 1 à 7 , obtenu pour chaque paire d'images par les 40 adultes. L'écart type est également indiqué.

En résumé sur le site Web, apparaissent, pour chaque association, les pourcentages moyens d'identification et de justifications taxonomiques, thématiques et perceptives évalués entre 3 et 8 ans, ainsi que le score moyen de force d'association testé entre 4 à 8 ans et celui de similarité perceptive évalué par des adultes.

\section{Tâche de production d'exemplaire : épreuve verbale}

Depuis les premiers travaux de Rosch (Rosch, 1973, 1975, 1978; Rosch \& Mervis, 1975; Rosch et al., 1976), l'existence des phénomènes de 
typicalité est bien connue. Certains exemplaires d'une catégorie seraient de meilleurs représentants que d'autres (le chien, par exemple, apparaît comme un membre plus représentatif de la catégorie animal que la tortue). Selon Rosch et Mervis (1975), les exemplaires les plus typiques possèdent ainsi de nombreuses propriétés partagées par la plupart des membres de la catégorie, contrairement aux atypiques qui ont des propriétés communes à peu de membres.

Les procédures utilisées pour évaluer la typicalité sont généralement des tâches de jugement de représentativité ou des épreuves de production d'exemplaires. Selon Hampton (1997), le jugement de typicalité réclame la considération attentive du degré de similarité entre un exemplaire et le reste de la catégorie et reflète la structure de l'information apprise. La tâche de production d'exemplaires, quant à elle, équivalente à un amorçage de la mémoire sémantique par le nom de la catégorie, fait appel à des processus de recherche et de rappel et reflète l'accessibilité des exemplaires. La corrélation élevée entre les 2 mesures provient du fait que les membres typiques tendent aussi à être les plus communément rencontrés et donc les plus accessibles (Hampton, 1997). Ces mesures jouent un rôle dans de nombreuses tâches comme par exemple la décision catégorielle, l'amorçage ou l'inférence inductive, ainsi que, comme l'a montré récemment Dell'Aqua (2003), la dénomination de l'objet: les objets considérés comme les plus typiques de leur catégorie sont dénommés plus vite que les moins typiques. Nous avons choisi la tâche de production d'exemplaires dans laquelle l'ordre de production s'expliquerait principalement par la typicalité. La mesure, dans ce cas, correspond à la fréquence moyenne dans le groupe de sujets des 5 premiers exemplaires produits (comme chez Cordier, 1980)

Matériel Pour éviter des interférences entre des catégories appartenant à la même hiérarchie catégorielle, 5 listes ont dû être constituées, comprenant chacune 8 ou 10 catégories. Nous avons voulu évaluer la typicalité des exemplaires sur deux types de catégories, des catégories taxonomiques surordonnées (par exemple « dis-moi toutes les fournitures scolaires que tu connais", "dismoi tous les animaux que tu connais »), et des catégories taxonomiques contextualisées (« dis-moi tout ce que l'on mange au petit-déjeuner»).

Population La passation étant longue, 40 enfants seulement à ce jour ont été évalués sur chaque liste à chaque âge, de 3 à 8 ans en moyenne, soit 1220 enfants en tout.

Procédure Chaque enfant est confronté à une seule liste de 8 ou 10 catégories. On lui demande de citer en une minute tous les exemplaires qu'il connaît à partir du nom d'une catégorie (terme inducteur). Lorsque l'enfant ne donne aucune réponse ou dit « je ne sais pas », aucune aide n'est apportée, l'expérimentateur passe à la catégorie suivante. Les catégories sont contrebalancées d'un enfant à l'autre pour une même liste. 
Critères d'analyse: score de typicalité Le score de typicalité est le pourcentage d'enfants d'un âge donné ayant mentionné l'exemplaire désigné parmi les 5 premiers exemplaires produits.

Il faut cependant rester prudent quant à l'interprétation des données normatives. En effet, Poitou (2000) pense que, lorsque l'on prend en compte les 4 premiers rangs de citation des exemplaires, on prend le risque que les exemplaires nommés en dernier soient en relation, non pas avec la catégorie énoncée (le terme inducteur), mais bien avec l'élément énoncé juste avant.

\section{INTERROGATION DE LA BASE}

BD2I est accessible sur le Web à l'adresse suivante: http://www.upmfgrenoble.fr/Banque_images/.

Elle se présente sous la forme d'une base de données informatisée, interrogeable à partir de multiples clés d'entrée. L'utilisateur peut choisir ses critères de recherche dans la page "recherche». Il peut obtenir des images d'objets selon leur appartenance catégorielle (taxonomique, thématique), sous-catégorielle (ex: mammifères après avoir choisi animaux dans la catégorie taxonomique des animaux), selon les normes relatives aux images isolées (ex sélectionner les images dénommées par $80 \%$ des enfants de 3 ans) ou associées (ex sélectionner les associations justifiées de façon taxonomique par $50 \%$ des enfants de 5 ans).

$\mathrm{Au}$ cours d'une session d'interrogation, l'utilisateur peut mettre les images sélectionnées dans "un panier de stockage » en vue de les enregistrer (avec les données normatives associées) ou les imprimer pour construire ses protocoles expérimentaux. Une aide en ligne guide l'utilisateur dans sa consultation et lui présente les fonctionnalités offertes (recherche, sauvegarde, etc.) ainsi que tous les détails de la procédure et des calculs de norme indiqués dans cet article.

\section{CONCLUSION}

Cet article présente la première base de données informatisée d'images fournissant des normes chez l'enfant français de 3 à 8 ans sur la connaissance de l'objet et les relations entre objets. L'objectif principal de cette base est de faciliter la construction de matériel expérimental en vue d'étudier 
et de comparer les performances des enfants français de 3 à 8 ans avec les données d'autres populations linguistiques et développementales (enfants plus âgés et adultes). Grâce à ces normes, il sera possible de mieux comprendre les processus cognitifs, tels que la catégorisation ou la flexibilité catégorielle. En contrôlant, à différents âges, à la fois l'identification des objets individuels et la reconnaissance correctement justifiée de toutes les associations proposées, nous pourrons déterminer comment évoluent les capacités conceptuelles de l'enfant.

BD2I présente bien sûr un grand intérêt pour l'étude du processus de dénomination d'image. Nos travaux à partir des données de la base confirment les résultats des études normatives déjà publiées et les complètent par les performances des enfants plus jeunes: les enfants de 3 et 4 ans produisent plus de réponses du type "ne connaît pas l'objet» et plus de réponses alternatives que les 6-8 ans. Le score d'accord de dénomination mesuré à partir de l'indice statistique $H$ rend compte de ces réponses alternatives mais se base sur le nom modal et ne tient pas compte des réponses du type "ne connaît pas l'objet» (Snodgrass \& Vanderwart, 1980). Or, lorsqu'un nom est donné par la majorité des participants, il ne correspond pas toujours au nom attendu par l'expérimentateur. Par exemple, la jambe (item 134) n'est dénommée que par $28 \%$ des enfants de 3 ans, qui produisent en majorité la réponse "pied». Ces réponses majoritaires mais erronées nous ont conduit à préconiser, lors de la constitution de normes, une mesure de la performance en dénomination en se basant sur le nom attendu plutôt que sur le nom majoritairement donné par les enfants (Cannard et al., 2005).

L'analyse des erreurs de dénomination en fonction des domaines a permis quant à elle, d'évaluer certains aspects du modèle différentiel de développement des concepts d'objet (Bonthoux et al., 2004). Les erreurs sémantiques fonctionnelles sont beaucoup plus nombreuses pour les objets fabriqués que naturels, ce qui confirme l'importance des propriétés fonctionnelles dans la construction de ce type de concept (Scheuner \& Bonthoux, 2004). En revanche le nombre des erreurs sémantiques visuelles ne diffère pas d'un domaine à l'autre, ce qui peut suggérer un rôle équivalent de la similarité dans les deux domaines.

Excepté pour quelques images dénommées dès 3 ans par $100 \%$ des participants (un tel taux de réussite signifie que le nom a sans doute été acquis beaucoup plus tôt), la base permet d'obtenir l'âge d'acquisition objectif des noms de chaque image ${ }^{1}$. Connaissant l'impact de ce facteur sur les

\footnotetext{
${ }^{1}$ Excepté celles pour lesquelles plusieurs noms étaient acceptés (cf. critères de dépouillement de la tâche de dénomination d'objet)
} 
processus de rappel et de production lexicale aussi bien chez les sujets sains que pathologiques, cette information est tout à fait fondamentale. De plus, corrélées avec les normes de production catégorielle, les données sur l'âge d'acquisition permettront de mieux appréhender la diversité du vocabulaire chez l'enfant en fonction des catégories sémantiques mais aussi la familiarité et la typicalité des exemplaires de chaque catégorie.

Par ailleurs, ce travail est susceptible d'aider aussi les enseignants d'écoles maternelles et primaires à choisir les entités faisant l'objet de séquences éducatives dans les domaines du langage et de la classification par exemple.

\section{BIBLIOGRAPHIE}

Alario, F.X., \& Ferrand, L. (1999). A set of pictures standardized for French: Norms for name agreement, image agreement, familiarity, visual complexity, image variability, and age of acquisition. Behavior Research Methods, Instruments and Computers, 31(3), 531-552.

Baldwin, D.A. (1992). Clarifying the role of shape in children's taxonomic assumptions. Journal of Experimental Child Psychology, 54, 392-416.

Barry, C., Hirsh, K.W., Johnston, R.A., \& Williams, C.L. (2001). Age of acquisition, word frequency, and the locus of repetition priming of picture naming. Journal of $\mathrm{Me}$ mory and Language, 44, 350-375.

Barry, C., Morrison, C.M., \& Ellis, A.W. (1997). Naming the Snodgrass and Vanderwart pictures: Effects of age of acquisition, frequency and name agreement. Quarterly Journal of Experimental Psychology: Human Experimental Psychology, 50A(3), 560-585.

Bates, E., Burani, C., D’Amico, S., \& Barca, L. (2001). Word reading and picture na- ming in Italian. Memory and Cognition, 29(7), 986-999.

Bates, E., D’Amico, S., Jacobsen, T., Szekely, A., Andonova, E., Devescovi, A., et al. (2003). Timed picture naming in seven languages. Psychonomic Bulletin Review, 10(2), 344-380.

Berman, S., Friedman, D., Hamberger, M., \& Snodgrass, J.G. (1989). Developmental picture norms: Relationships between name agreement, familiarity, and visual complexity for child and adult ratings of two sets of line drawings. Behavior Research Methods, Instruments and Computers, 21(3), 371-382.

Blaye, A., Bernard-Peyron, V., \& Bonthoux, F. (2000). Au-delà des conduites de catégorisation: le développement des représentations catégorielles entre 5 et 9 ans. Archives de Psychologie, 68, 59-82.

Blaye, A., \& Bonthoux, F. (2001). Thematic and taxonomic relations in preschoolers: the development of flexibility in categorization choices. British Journal of Developmental Psychology, 19, 395-412. 
Bonin, P., Chalard, M., Méot, A., \& Fayol, M. (2001). Age-of-acquisition and word frequency in the lexical decision task: Further evidence from the French language. Cahiers De Psychologie Cognitive Current Psychology of Cognition, 20(6), 401-443.

Bonin, P., Chalard, M., Méot, A., \& Fayol, M. (2002). The determinants of spoken and written picture naming latencies. British Journal of Psychology, 93 Part 1, 89-114.

Bonin, P., Fayol, M., \& Chalard, M. (2001). Age of acquisition and word frequency in written picture naming. The Quarterly Journal of Experimental Psychology, 54(2), 469-489.

Bonthoux, F., Berger, C., \& Blaye, A. (2004). Naissance et développement des concepts chez l'enfant. Catégoriser pour comprendre. Paris : Dunod.

Bonthoux, F., \& Blaye, A. (1999). Multiple categorical representations for the same object: The development of representational flexibility in children, 1st bisontine conference for conceptual and linguistic development in the child aged from 1 to 6 years. Besançon, France.

Brysbaert, M., Van Wijnendaele, I., \& De Deyne, S. (2000). Age-of-acquisition effects in semantic processing tasks. Acta Psychologica, 104(2), 215-226.

Cannard, C., Blaye, A., Scheuner, N., \& Bonthoux, F. (2005). Picture naming in 38 year-old French Children: Methodological considerations for name agreement. Behavior Research Methods Instruments and Computers, 37(3), 417-425.

Caroll, J.B., \& White, M.N. (1973). Age-ofAcquisition Norms for 220 picturable Nouns. Journal of Verbal Learning and Verbal Behavior, 12(5), 563-576.

Chainay, H., Rosenthal, V., \& Goldblum, M.C. (1998). Normes de dénomination de 315 images en couleur et en noir et blanc. Revue de Neuropsychologie, 8(2), 179-239.

Chalard, M., Bonin, P., Méot, A., Boyer, B., \& Fayol, M. (2003). Objective age-ofacquisition (AoA) norms for a set of 230 object names in French: Relationships with psycholinguistic variables, the English data from Morrison et al. (1997), and naming latencies. European Journal of Cognitive Psychology, 15(2), 209-245.

Chemlal, S. (2000). Scripts et développement des connaissances catégorielles. Psychologie Française, 45(2), 131-140.

Clark, J.M., \& Johnson, C.J. (1994). Retrieval mechanisms in the development of instance and superordinate naming of pictures. J Exp Child Psychol, 57(3), 295-326.

Cordier, F. (1980). Gradients de prototypie pour cinq catégories sémantiques. Psychologie Française, 25, 211-219.

Cuetos, F., Ellis, A.W., \& Alvarez, B. (1999). Naming times for the Snodgrass and Vanderwart pictures in Spanish. Behavior Research Methods Instruments and Computers, 31(4), 650-658.

Cycowicz, Y.M., Friedman, D., Rothstein, M., \& Snodgrass, J.G. (1997). Picture naming by young children: Norms for name agreement, familiarity, and visual complexity. Journal of Experimental Child Psychology, 65(2), 171-237.

Daehler, M.W., Lonardo, R., \& Bukatko, D. (1979). Matching and equivalence judgments in very young children. Child Development, 50, 170-179.

D’Amico, S., Devescovi, A., \& Bates, E. (2001). Picture naming and lexical access in Italian children and adults. Journal of Cognition and Development, 2(1), 71-105.

De La Haye, F. (2003). Normes d'associations verbales chez des enfants de 9, 10 et 11 ans et des adultes. L'Année Psychologique, 2003(103), 109-130.

Dell' Acqua, R., Lotto, L., \& Job, R. (2000). Naming times and standardized norms for the Italian PD/DPSS set of 266 pictures: Direct comparisons with American, English, French, and Spanish published databases. Behavior Research Methods Instruments and Computers, 32(4), 588-615.

Ellis, A.W., \& Morrison, C.M. (1998). Real age-of-acquisition effects in lexical retrieval. Journal of Experimental Psychology: 
Learning, Memory, and Cognition, 24(2), 515-523.

Fenson, L., Cameron, M.S., \& Kennedy, M. (1988). Role of perceptual and conceptual similarity in category matching at age two years. Child Dev, 59(4), 897-907.

Fenson, L., Vella, D., \& Kennedy, M. (1989). Children's knowledge of thematic and taxonomic relations at two years of age. Child Dev, 60(4), 911-919.

Ferrand, L., \& Alario, F.X. (1998). Normes d'associations verbales pour 366 noms d'objets concrets. L'année Psychologique, 98, 659-709.

Gerhand, S., \& Barry, C. (1999). Age of acquisition, word frequency, and the role of phonology in the lexical decision task. $M e$ mory and Cognition, 27(4), 592-602.

Gilhooly, K.J., \& Gilhooly, L.M. (1980). The validity of age-of-acquisition ratings. British Journal of Psychology, 71, 105-110.

Gilhooly, K.J., \& Hay, D. (1977). Imagery, concreteness, age-of-acquisition, familiarity and meaningfulness values for 205 five-letter words having single solution anagrams. Behavior Research Methods and Instrumentation, 9, 12-17.

Golinkoff, R.M., Shuff-Bailey, M., Olgin, R., \& Ruan, W. (1995). Young children extend novel words at the basic level: Evidence for the principle of categorical scope. Developmental Psychology, 31, 497-507.

Hampton, J.A. (1997). Associative and similarity-based processes in categorization decisions. Memory and Cognition, 25, 625-640.

Humphreys, G.W., Riddoch, M.J., \& Quinlan, P.T. (1988). Cascade processes in picture identification. Cognitive Neuropsychology, 5(1), 67-103.

Imai, M., Gentner, D., \& Uchida, N. (1994). Children's theories of word meaning: The role of shape similarity in early acquisition. Cognitive Development, 9, 45-75.

Johnson, C.J. (1992). Cognitive components of naming in children: effects of re- ferential uncertainty and stimulus realism. J Exp Child Psychol, 53(1), 24-44.

Johnson, C.J., Paivio, A., \& Clark, J.M. (1996). Cognitive components of picture naming. Psychological Bulletin, 120(1), 113-139.

Kremin, H., Akhutina, T., Basso, A., Davidoff, J., De Wilde, M., Kitzing, P., et al. (2003). A cross-linguistic data bank for oral picture naming in Dutch, English, German, French, Italian, Russian, Spanish, and Swedish (PEDOI). Brain and Cognition, 53(2), 243-246.

Kremin, H., Hamerel, M., Dordain, M., De Wilde, M., \& Perrier, D. (2000). Age of acquisition and name agreement as predictors of mean response latencies in picture naming of french adults. Brain and Cognition, 43(1-3), 286-291.

Levelt, W.J. (1989). Speaking: From intention to articulation. Cambridge, MA: MIT Press.

Lin, E.L., \& Murphy, G.L. (2001). Thematic relations in adults'concepts. Journal of Experimental Psychology: General, 130, 3-28.

Lucariello, J., Kyratzis, A., \& Nelson, K. (1992). Taxonomic knowledge: What kind and when? Child Development, 63, 978-998. Markman, E.M., \& Hutchinson, J.E. (1984). Children's sensitivity to constraints on word meaning: taxonomic vs thematic relations. Cognitive Psychology, 16, 1-27.

Martein, R. (1995). Norms for name and concept agreement, familiarity, visual complexity and image agreement on a set of 216 pictures. Psychologica Belgica, 35(4), 205-225.

Morrison, C.M., Chappell, T.D., \& Ellis, A.W. (1997). Age of acquisition norms for a large set of object names and their relation to adult estimates and other variables. Quarterly Journal of Experimental Psychology: Human Experimental Psycho$\log y, 50 A(3), 528-559$.

Morrison, C.M., \& Ellis, A.W. (1995). The roles of word frequency and age of acquisition in word naming and lexical decision. 
Journal of Experimental Psychology: Learning Memory and Cognition, 21, 116-133.

Morrison, C.M., Ellis, A.W., \& Quinlan, P.T. (1992). Age of acquisition, not word frequency, affects object naming, not object recognition. Memory and Cognition, 20(6), 705-714.

Osborne, J.G., \& Calhoun, D.O. (1998). Themes, taxons, and trial types in children's matching to sample. Methodological considerations. Journal of Experimental Child Psychology, 68, 35-50.

Pind, J., Jonsdottir, H., Gossurardottir, H., \& Jonsson, F. (2000). Icelandic norms for the Snodgrass and Vanderwart (1980) pictures: Name and image agreement, familiarity, and age of acquistion. Scandinavian Journal of Psychology, 41(1), 4148.

Poitou, J. (2000). Prototypes, saillance et typicalité. Terminologies Nouvelles, 21, 1-15.

Rosch, E. (1973). Natural categories. Cognitive Psychology, 4, 328-350.

Rosch, E. (1975). Cognitive representations of semantic categories. Journal of Experimental Psychology: General, 104, 192-233.

Rosch, E. (1978). Principles of categorization. In E. Rosch \& B. B. Lloyd (Eds.), Cognition and categorization (pp. 27-48). Hillsdale, NJ: Erlbaum.

Rosch, E., \& Mervis, C.B. (1975). Family resemblances: studies in the internal structure of categories. Cognitive Psychology, 7, 573-605.

Rosch, E., Mervis, C.B., Gray, W., Johnson, D., \& Boyes-Braem, P. (1976). Basic objects in natural categories. Cognitive Psychology, 3, 382-439.

Sanfeliu, M.C., \& Fernandez, A. (1996). A set of 254 Snodgrass-Vanderwart pictures standardized for Spanish: Norms for name agreement, image agreement, familiarity, and visual complexity. Behavior Research Methods, Instruments and Computers, 28(4), 537-555.

Scheuner, N., \& Bonthoux, F. (2004). La construction des catégories surordonnées chez l'enfant: utilisation différentielle des indices perceptif et contextuel dans le domaine du vivant et du non vivant. Bulletin de Psychologie, 57, 105-109.

Scheuner, N., Bonthoux, F., Cannard, C., \& Blaye, A. (2004). The role of associative strength and conceptual relations in matching tasks in 4- and 6-year-old children. International Journal of Psychology, 39(4), 290-304.

Sell, M.A. (1992). The development of children's knowledge structures: Events, slots, and taxonomies. Journal of Child Language, 19, 659-676.

Smiley, S.S., \& Brown, A.L. (1979). Conceptual preference for thematic or taxonomic relations: A non monotonic age trend from preschool to old age. Journal of Experimental Child Psychology, 28, 249-257.

Snodgrass, J.G., \& Vanderwart, M. (1980). A standardized set of 260 pictures: Norms for name agreement, image agreement, familiarity, and visual complexity. Journal of Experimental Psychology: Human Learning and Memory, 6(2), 174-215.

Szekely, A., D’Amico, S., Devescovi, A., Federmeier, K., Herron, D., Iyer, G., et al. (2003). Timed picture naming: Extended norms and validation against previous studies. Behavior Research Methods Instruments and Computers, 35(4), 621633.

Van Schjagen, I., Tamsa, N., Bruggemann, F., Jackson, J.L., \& Michon, J.A. (1983). Names and norms for pictures. Nederlands Tijdschrift voor de Psychologie, 38, 236-241. Vitkovitch, M., \& Tyrrell, L. (1995). Sources of disagreement in object naming. Quarterly Journal of Experimental Psychology: Human Experimental Psychology, 48A(4), 822-848.

Walsh, M., Richardson, K., \& Faulkner, D. (1993). Perceptual, thematic and taxonomic relations in children's mental representations: Responses to triads. European Journal of Psychology of Education, 8, 85-102.

Waxman, S.R., \& Namy, L.L. (1997). Challenging the notion of a thematic preference 
in young children. Developmental Psycho$\log y, 33,555-567$.

Wiegel-Crump, C.A., \& Dennis, M. (1986). Development of word-finding. Brain and Language, 27(1), 1-23.
Wisnieski, E.J., \& Bassok, M. (1999). What makes a man similar to a tie? Stimulus compatibility with comparison and integration. Cognitive Psychology, 39, 208238. 